\title{
OBSERVATIONS ON THE EPIDEMIOLOGY OF STREPTOCOCCAL PHARYNGITIS AND THE RELATION OF STREPTOCOCCAL CARRIERS TO THE OCCURRENCE OF OUTBREAKS ${ }^{1}$
}

\author{
By ANN G. KUTTNER AND ELMA KRUMWIEDE
}

(From Irvington House, Iroington-on-Hudson, N. Y.)

(Received for publication June 7, 1943)

One of the most difficult problems confronting physicians interested in the control of infectious diseases is that of "post-infection" and "healthy" carriers. The epidemiology of diseases associated with streptococci is particularly baffling because of the frequent occurrence of these microorganisms in the throats of normal individuals. It is only since the development of serological methods for differentiating hemolytic streptococci into groups and types by Lancefield $(1,2)$ and Griffith (3) that accurate epidemiological studies of streptococcal diseases have become possible. Although several surveys of epidemic scarlet fever and streptococcal pharyngitis, in which the strains were identified by these serological methods, have been published, most of these reports cover mainly the period of the outbreak and do not include data on preepidemic carrier rates. It seemed worthwhile, therefore, to report observations covering a 6-year period on the incidence of carriers of various Goup A streptococci and their relation to the occurrence of outbreaks of streptococcal pharyngitis among groups of rheumatic children under close observation in a sanatorium.

\section{LITERATURE}

Since most observers did not have the opportunity of studying pre-epidemic carrier rates of Group A streptococci, no attempt will be made to review the literature completely.

Although the observations of Bloomfield and Felty were made before methods for the serological differentiation of streptococci were available, these authors described many of the epidemiological features characteristic of outbreaks of streptococcal pharyngitis $(4,5)$. No rise in the carrier rate occurred at the time that cases began to appear. The infecting dose was

1 These studies were aided by a grant from The Commonwealth Fund. usually acquired as the result of prolonged close contact and the susceptibility of different subjects varied greatly. According to these observers, individuals did not become "contact" carriers unless infection had occurred. This point of view has been reaffirmed in a recent article by Bloomfield and Rantz (6).

Dudley studied the incidence of scarlet feverand diphtheria in a boys' school (7). No cases of scarlet fever or diphtheria developed among the 100 day pupils, whereas both diseases were prevalent among the 300 boarding pupils. This difference was explained by Dudley in the following way: the natural defenses of the body are able to destroy small numbers of the infecting agent. When contact is prolonged, however, the velocity of destruction may be less than the rate at which the pathogenic bacteria are acquired, so that eventually the minimal infecting dose is reached.

In studies of epidemics of scarlet fever occurring in Rumania, Schwentker was able to obtain data on pre-epidemic carrier rates (8). He found that a definite rise in the carrier rate of the epidemic type preceded outbreaks. In the opinion of this observer, a marked increase in the number of carriers in a community carrying a single type of Group A streptococci may be used as an indication that an epidemic is imminent.

\section{MATERIAL}

The type of institution and the bacteriological procedures have been previously described (9). The relationship of the outbreaks of streptococcal upper respiratory infections to rheumatic relapses has also been reported $(9,10)$. From an epidemiological point of view, the following procedures and data are important. With only occasional exceptions, no new children were admitted during the winter months, i.e., from November or December until the end of May or the middle of June. Each year the same 108 children, ranging in age from 7 to 15 years, remained in the institution for 6 months or longer and thus constituted essentially a "closed" colony.

The children slept in 5 wards of 18 to 24 beds each and 
ate in one large dining room. They attended school in 2 rooms in the same building. The 66 girls were divided into 3 and the 42 boys into 2 recreational groups. Children were usually admitted in groups of $\mathbf{8}$ and were isolated for 48 hours. During this time 2 throat cultures were taken to determine the presence of Group A streptococci. Thereafter, weekly throat cultures were taken routinely on every child throughout the year. When Group A streptococci were first isolated from a child, who had no complaints or clinical symptoms of an upper respiratory infection, the following tests were used to determine the presence of subclinical infection: leukocyte counts, erythrocyte sedimentation rates, and determinations of antistreptolysin $\mathbf{O}$ titers. If the laboratory findings remained unchanged, the child was considered to be a "healthy" carrier. In the case of known Group A streptococcus carriers, when a different colony form was observed on a routine throat culture plate, suggesting a possible change in type, similar laboratory procedures were employed to rule out the possibility of subclinical infection. Throat cultures were taken on 2 successive days on all children who showed symptoms of any kind. Nasal cultures were taken only when clinical evidence of nasal infection was present.

Attempts were made to group and type all hemolytic streptococci isolated. The details of the methods used have been published elsewhere (11). Streptococci isolated from known carriers were checked serologically once a month and more of ten when clinical or laboratory evidence suggested a possible change of type.

\section{Chassification of carriers}

For the purposes of this study, carriers of Group A streptococci are divided into 3 categories: "post-infection," "contact," and "healthy." "Post-infection" carrier refers to children who develop streptococcal pharyngitis while under observation and then become carriers. "Contact" is used to designate individuals in the institution who, without developing clinical or laboratory evidence of infection, acquire streptococci either from other children with streptococcal upper respiratory infections or from known carriers. The term "healthy" carrier is restricted to children harboring streptococci on admission or shortly thereafter since it was not known whether they were "postinfection" or "contact" carriers.

During each year, the weekly incidence of all streptococcus carriers in the group was determined. Carriers were classified as temporary if they harbored hemolytic streptococci less than 2 months, and as chronic if for longer periods. Chronic carriers were further classified as intermittent or consistent. Observations based on 1 or 2 cultures gave an inaccurate picture of the number of carriers in the community. Not only may streptococci from intermittent carriers be missed, but even fairly consistent carriers occasionally fail to show these microorganisms. Weekly throat cultures obtained from chronic carriers may be negative for as long as 12 weeks and then become positive. Furthermore, irrespective of whether the carrier had clinical signs of a "common cold," marked variations in the number of streptococci isolated at different times from the same child were observed. In our opinion, therefore, the assumption that a particular carrier is not a possible source of danger, because 2 or 3 cultures yield only a few colonies of streptococci, is unwarranted.

\section{EPIDEMIOLOGICAL FINDINGS}

During the winters of 1937-38 and 1942-43, only a small number of cases of streptococcal pharyngitis occurred. The spread of infection during these 2 years differed from that observed during the 4 intervening years when the incidence of streptococcal pharyngitis was greater. The 2 years $1937-38$ and $1942-43$ are therefore grouped together and will be described later. The 4 winter seasons from 1938 to 1942 , when the incidence of streptococcal upper respiratory infections was fairly high, are discussed first.

\section{A. Major outbreaks of streptococcal pharyngitis due to types not previously in the community}

\section{Outbreak of streptococcal pharyngitis due to type 4, February to June 1939}

Group $A$ streptococcus carriers in the community on February 1, 1939. The 108 patients included 66 "new" (i.e., those admitted during the summer and fall months) and 42 "old" children (i.e., those who resided in the institution during the previous winter). Twenty-one of the 66 "new" children were "healthy" carriers of Group A streptococci. The following types were represented: types $5,12,17,18,19,23,28,29$, and 32 , and 11 strains of undetermined types. In some instances, when a child became a carrier several weeks after admission, it was impossible to tell whether he was an intermittent "healthy" or a "contact" carrier who had acquired the strain from another child carrying the same type or from some unknown source, such as visitors or staff.

The carrier rate fell during the course of the winter so that by February 1 , only 8 of the 21 "new" children who had been "healthy" carriers of Group A streptococci still harbored these microorganisms: 4 carried, respectively, types 5 , $17,19,29$, and the others, strains of undetermined types. Three of the 42 "old" children were chronic "healthy" carriers: one girl carried type 18 for more than 2 years; 2 other children carried streptococci of undertermined types for 21 and 22 months, respectively. 
Thus, in February 1939, 11 of the 108 children, or 10 per cent, were carriers of Group A streptococci.

Outbreak of pharyngitis due to type 4 streptococcus, February 4 to May 15, 1939. On February 4, a girl developed acute pharyngitis due to type 4 streptococcus, a type not previously present in the community. It was thought possible that the child contracted the infection from her parents who had visited her 2 days before symptoms appeared. Three other girls developed pharyngitis due to type 4 streptococcus during February. The infection spread to the boys during March and reached its peak with 17 patients: 8 girls and 9 boys. In April and May, the incidence declined to 6 and 5 cases, respectively, and was confined mainly to the girls ( 9 girls and 2 boys). Thus, during 4 months, a total of 32 cases occurred among the 108 children.

The monthly incidence of cases of type 4 streptococcal pharyngitis, type 4 "contact" carriers, and the carrier rate of miscellaneous Group A streptococci are presented in Figure 1.

No type 4 carriers were present in the community in February 1939 when the first 4 cases of pharyngitis due to this type appeared. The incidence of type 4 "contact" carriers rose

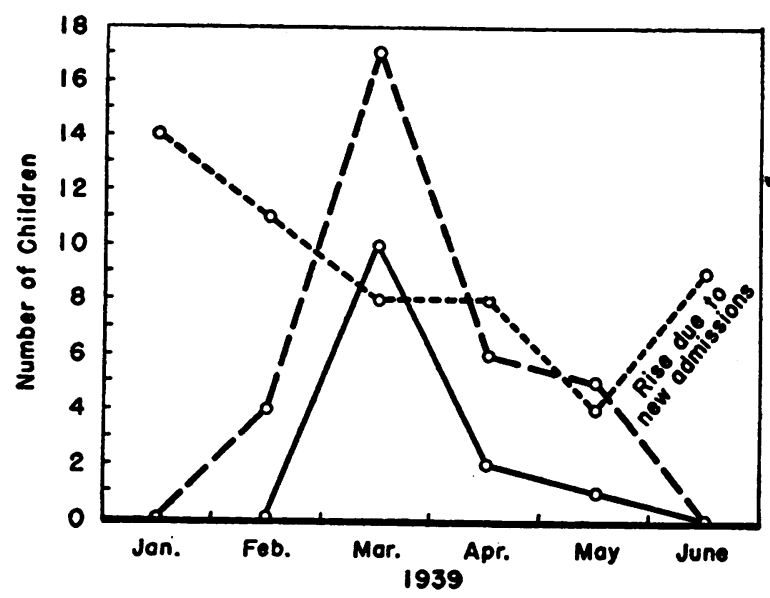

Fig. 1. Monthly Incidence of 32 Cases of Type 4 Pharyngitis, Type 4 Carriers, and Carriers of Miscellaneous Group A Streptococci

$\odot---$ Type 4 cases

$\odot-$ Type 4 "contact" carriers

○- - - - "Healthy" carriers of miscellaneous Group A streptococci sharply in March at the time when the greatest number of cases occurred and fell rapidly as the incidence of infections decreased. The carrier rate of other types of Group A streptococci fell gradually during the course of the outbreak.

In addition to the cases of upper respiratory infections due to type 4 streptococcus during March, 6 children developed pharyngitis due to Group A streptococci of undetermined type but with similar cultural characteristics. It was thought possible, therefore, that they might belong to one serological type. Attempts to produce agglutinating and precipitating sera by immunizing rabbits with one of these cultures were, however, unsuccessful and the serological identity of these 6 strains was not proved.

The remaining 56 children in the institution escaped streptococcal pharyngitis and did not become "contact" carriers of type 4 streptococcus.

\section{Outbreak of streptococcal pharyngitis due to type 27, October 1939 to May 1940}

Group A streptococcus carriers in the community on November 1, 1939. Sixty-seven of the 108 patients, followed during this winter, were "new" and 41, "old." Twenty-one of the 67 "new" children were "healthy" carriers of Group A streptococci. The following types were represented: types $2,3,32,33$, and 39 , and 15 strains of undetermined types.

By November 1, only 6 of the 21 "healthy" carriers were still harboring streptococci: one child was carrying type 2 , one type 39 , and 4 , streptococci of undetermined types.

Between September 11 and November 1, 2 "new" and 4 "old" children became carriers of type 33 streptococcus, a strain introduced by a "new" boy admitted in June 1939. During the course of 4 months, this type became disseminated among 22 children without, except in one instance, causing symptoms.

In addition to the carriers of type 33 streptococcus, 5 of the "old" children were carrying type 4 streptococcous, the epidemic strain of the previous year. One "old" girl carried type 29 streptococcus until the middle of January. Neither of these two types showed a tendency to spread to "new" children.

Outbreak of pharyngitis due to type 27 strepto- 
coccus, November 2, 1939, to A pril 19, 1940. On October 31, a girl (G. C.) whose throat cultures had been consistently negative for Group A streptococci for 4 months, was sent to another hospital for tonsillectomy. She was readmitted on November 3, 2 days after operation, and had fever for 5 days after her return. Her throat cultures showed numerous type 27 streptococci, a type not previously present in the community. Another child, who had a tonsillectomy at the same hospital at the same time and who shared the same cubicle, failed to contract this infection.

Two weeks after the readmission of G. C., 3 other girls on the same ward developed pharyngitis due to type 27 streptococcus. During the next 6 months, 36 girls contracted this infection and 12 girls become "contact" carriers of type 27. In spite of the fact that 10 boys became "contact" carriers of the epidemic type, only 3 boys developed pharyngitis.

The monthly incidence of cases of type 27 streptococcal pharyngitis, type 27 "contact" carriers, type 33 streptococcus carriers, and carriers of miscellaneous Group A streptococci are presented in Figure 2.
The incidence of "contact" carriers of the epidemic-inducing type rose slowly compared with the incidence of cases: one child became a carrier in November when 5 cases appeared, and 2 in December when 8 further cases developed. The highest incidence of both "contact" carriers and cases occurred in January and then declined simultaneously. The carrier rate of type 33 streptococcus reached its peak in November. During December and January, both the miscellaneous Group A streptococcus carrier rate and that of type 33 were falling.

The remaining 46 children in the institution escaped streptococcal pharyngitis and did not become "contact" carriers of type 27 streptococcus.

\section{Outbreak of streptococcal pharyngitis due to type 15, October 1940 to February 1941}

Group $A$ streptococcus carriers in the community during the fall of 1940 . Seventy of the 108 patients followed during this winter were "new" and 38 "old." Nineteen of the 70 "new" children were "healthy" carriers of Group A streptococci. The following types were repre-

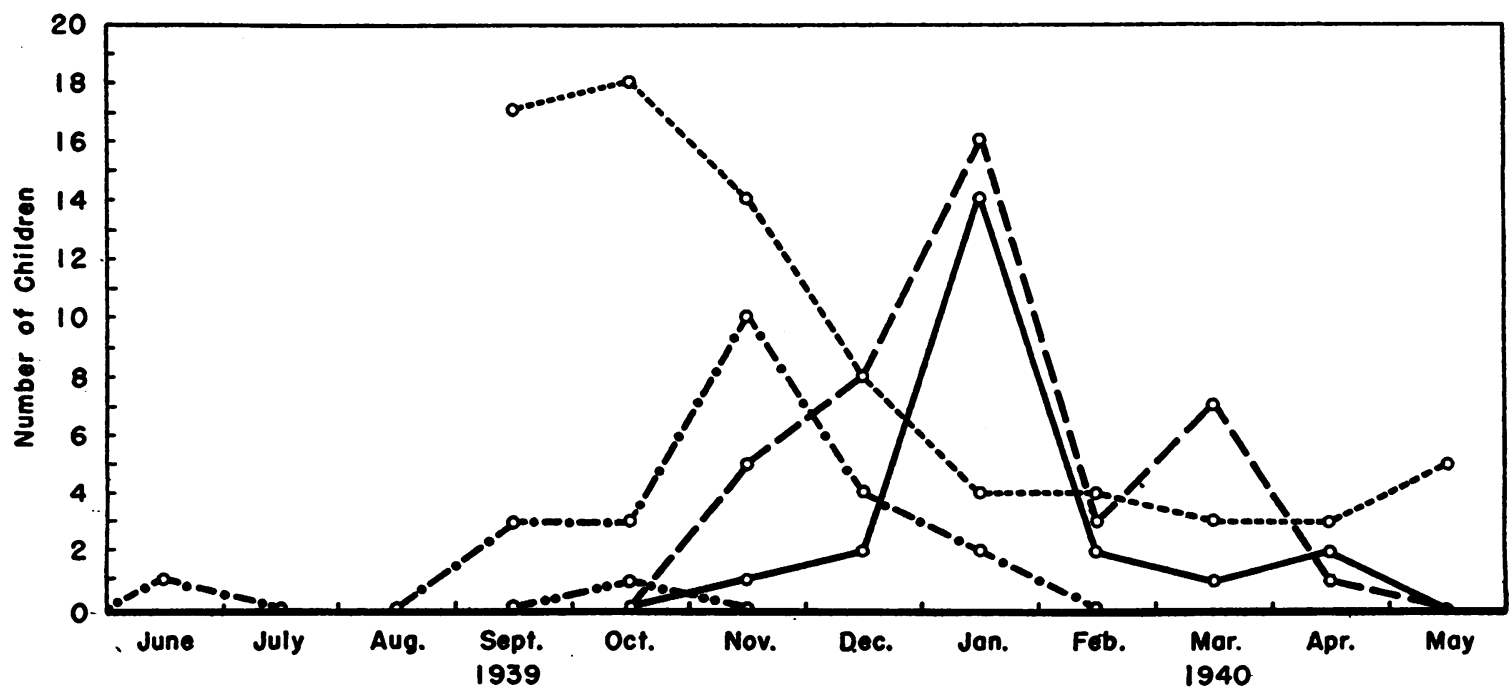

Fig. 2. Monthly Incidence of 40 Cases of Type 27 Pharyngitis, Type 27 Carriers, Type 33 Carriers, and Carriers of Misceilaneous Group a Streptococci

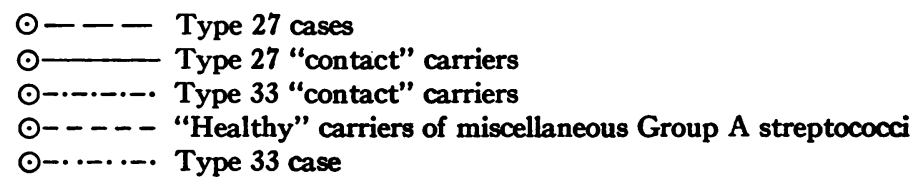


sented: types $2,6,12,13,17,26,27,33,34$, and 38 , and 4 strains of undetermined types. One boy carried both type 6 and type 18 .

Sporadic cases of streptococcal upper respiratory infections occurring in the fall of 1940 . Eleven cases due to 3 different types of streptococci, types 6,25 , and 27 , were observed during the fall of 1940. Between the middle of September and November 1st, 7 cases of type 27 pharyngitis occurred among the 70 "new" children who had not been in the institution the previous year when this type was epidemic. The upper respiratory symptoms in these 7 children were milder than those observed during the outbreak of the previous winter. The cultural characteristics of the type 27 streptococci, isolated from these cases, also differed from those of the strains, isolated during the previous epidemic: the capsules in India Ink preparations were smaller and the colonies less mucoid. One "new" child was found to be a type 27 carrier on admission. In September, 17 "old" children were still carrying type 27 , so that there were many possible sources of this strain to account for these 7 cases. One "new" girl became a "contact" carrier of type 27 , and harbored this strain for 6 months.

A single case of infection due to type 25 streptococcus occurred in September in a "new" boy, 2 months after admission. There were no known carriers of type 25 in the community at this time and the source of this strain was not determined. No other cases of infection due to type 25 developed; but 2 boys subsequently became temporary "contact" carriers of this type.

Two cases of type 6 streptococcal pharyngitis developed on one boys' ward where there were 2 "healthy" carriers of this type. The first case occurred in August and the second in September. In October, a third case due to type 6 occurred in a girl. Because the infection had jumped to one girls' ward, it was thought that an epidemic due to type 6 might be starting. Beginning October 18, therefore, daily prophylactic doses of sulfanilamide were given to half of the children in the institution. However, no further cases of pharyngitis due to type 6 streptococcus developed.

By the end of October, 17 of the 19 "new" children who were "healthy" carriers ceased to harbor hemolytic streptococci. The throat cultures of the boy carrying types 6 and 18 remained intermittently positive for both types for 8 months and another child carried streptococci of undetermined type for more than one year. Among the "old" children, one boy harbored type 34 streptococcus for 10 months. Four children were chronic "post-infection" carriers of type 27, the epidemic-inducing streptococcus of the previous year. Other carriers in the community at this time were the result of the 11 sporadic streptococcal infections which occurred from September until the end of October: 5 children were carrying type 27 streptococcus; 2, type 6; and one, type 25.

Outbreak of pharyngitis due to type 15 streptococcus, October 22, 1940 to January 29, 1941

Control group (i.e., children not receiving sulfanilimide). On October 17, 1940, a girl was admitted whose throat culture showed a single colony of type 34 streptococcus. A second culture, taken on the next day, showed no hemolytic streptococci. Five days after admission, a routine throat culture showed many type 15 streptococci but no type 34 . The source of the type 15 strain was not determined. Although throat cultures obtained from this child continued to show numerous colonies of type 15 streptococcus, she showed no clinical or laboratory evidence of infection. She was, therefore, considered to be a carrier.

A series of cases due to type 15, which had not been previously present, developed first among the girls and then spread to the boys. During the next 3 months, among the 54 children who were not receiving sulfanilamide, 21 girls and 9 boys contracted pharyngitis due to this type. One other child became a "contact" carrier of type 15 streptococcus.

Sulfanilamide treated group. In spite of a blood level of $2 \mathrm{mgm}$. per cent of sulfanilamide, one child developed pharyngitis due to type 15 streptococcus. Ten children who were receiving prophylactic sulfanilamide became "contact" carriers of the epidemic-inducing type.

The monthly incidence of 11 sporadic cases of streptococcal upper respiratory infections due to types 6,25 , and 27 , the outbreak of 31 cases of pharyngitis due to type 15 , the carrier rates of types $6,15,27$, and miscellaneous Group A 
streptococci in the untreated and sulfanilamide treated groups are presented in Figure 3.

There was only one type 15 streptococcus carrier in the community in October when the first 3 cases of type 15 streptococcal pharyngitis appeared. In November, when 8 further cases among the children in the control group developed, 4 children receiving sulfanilamide became "contact" carriers. In December, when the outbreak reached its peak and 12 untreated children contracted the infection, one child in the sulfanilamide group developed pharyngitis due to type 15 streptococcus and one became a "contact" carrier. In January, the number of cases appearing in the control group declined to 7. No further cases developed among the children receiving sulfanilamide, but 2 more children became "contact" carriers. The carrier rate of type 27 streptococcus and of miscellaneous Group A streptococci decreased at approximately the same rate in control and sulfanilamide-treated groups.

In February, the throat cultures of 37 , or 34 per cent, of the 108 children were positive for type 15 streptococcus. In spite of this large number of possible sources of infection, no new cases developed among the 24 children in the control group who had previously escaped infection.

\section{Outbreak of streptococcal pharyngitis due to type 36, October 1941 to March 1942}

Group $A$ streptococcus carriers in the community during the fall of 1941 . The group followed this winter included 73 "new" and 35 "old" patients. During September, 11 "new" children were "healthy" carriers of Group A streptococci. The following types were represented: types $1,2,4$, $5,14,18$, and 25 , and 3 strains of undetermined types. (Two children carried the same type, type 2.)

In July, a "new" girl contracted mild pharyngitis due to type 35 streptococcus, 3 weeks after admission, and then carried this type for one year. In August and September, 3 other children developed upper respiratory infections due to type 35 and 2 girls became "contact" carriers. Three "old" children were still carrying type 15 streptococcus, the epidemic strain of the previous year. Thus, during September, 17 of 108 chil-
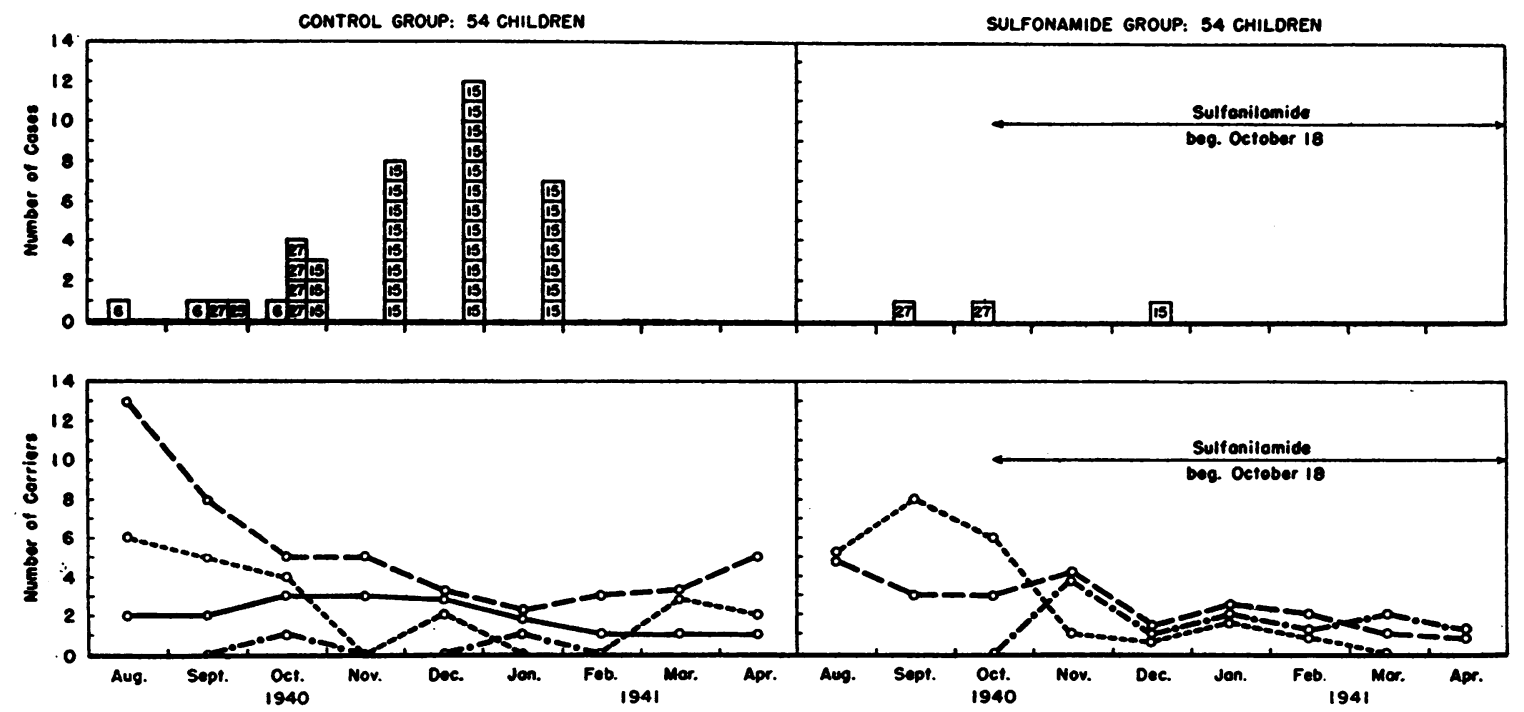

Fig. 3. Monthly Incidence of 10 Sporadic Cases of Pharyngitis Due to Types 6,"25, and 27, and an Outbreak of 30 Cases of Pharyngits Due to Type 15 Streptococcus
6 Type 6 cases
$\odot-$ Type 6 "post-infection" and "contact" carriers
25 Type 25 cases
$\odot-1-$ Type 27 "post-infection" and "contact" carriers
27 Type 27 cases
๑-.-.-... Type 15 "contact" carriers
15. Type 15 cases

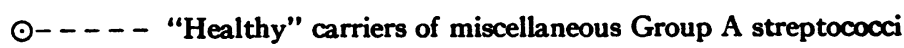




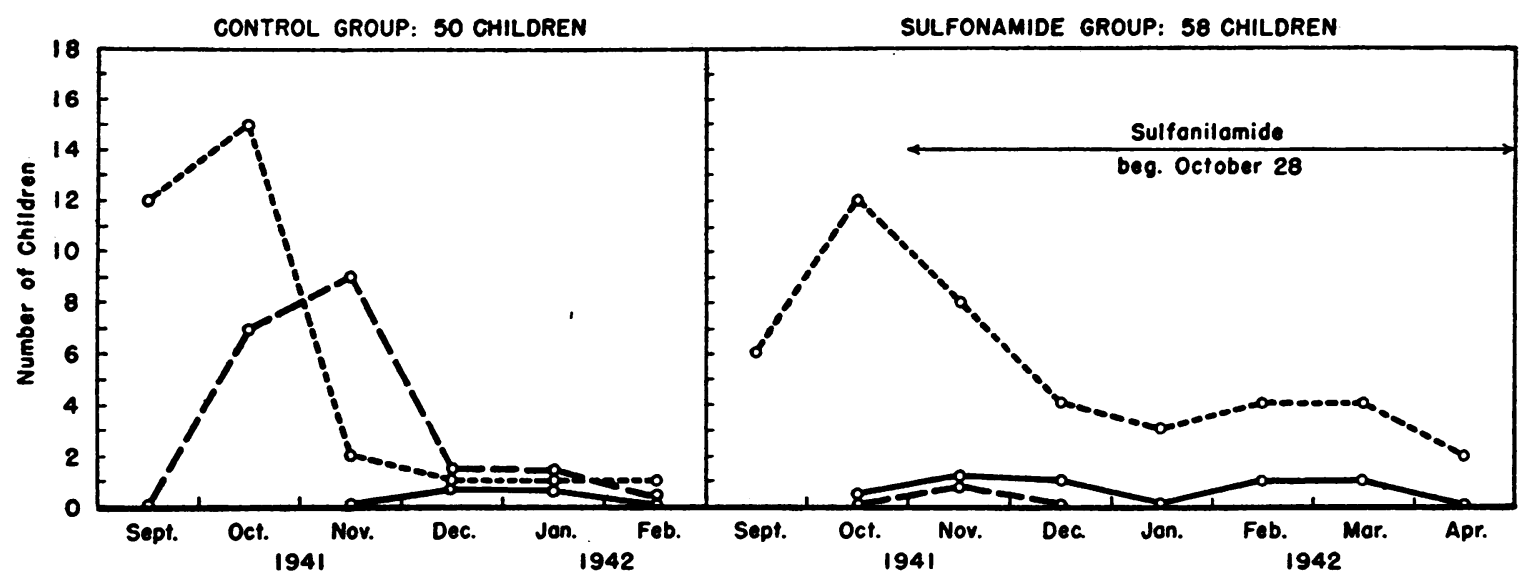

Fig. 4. Monthly Incidence of 18 Cases of Type 36 Pharyngitis, Type 36 Carriers, and Carriers of Miscellaneous Group A Streptococci

$\odot---$ Type 36 cases

$\odot$ Type 36 "contact" carriers

○- - - - "Healthy" carriers of miscellaneous Group A streptococci

dren were Group A streptococcus carriers. In October, the number of carriers rose to 27, due to the admission of "new" children who were carriers and to intermittent carriers whose throat cultures previously had shown streptococci but were negative in September. During November, the carrier rate dropped sharply and decreased still further in December and January.

Outbreak of pharyngitis due to type 36, October 26, 1941 to January 21, 1942. On October 21, a boy (H. B.) with slight coryza was admitted. His admission throat culture contained type 36 streptococcus, a type not previously present in the community. A series of cases of pharyngitis due to this strain developed, first among the boys and then spread to the girls. Beginning October 28, daily prophylactic doses of sulfanilamide or sulfadiazine were given to 58 children.

Control group. During the next 3 months, 9 boys and 8 girls among the 50 children serving as untreated controls contracted pharyngitis due to type 36. Two children became "contact" carriers of this type.

The remaining 30 children in the control group escaped streptococcal pharyngitis and did not become "contact" carriers of type 36.

Sulfonamide treated group. One boy developed pharyngitis due to type 36 . The blood level of the drug at this time was less than $1 \mathrm{mgm}$. per cent. Four children in this group became "contact" carriers of type 36.
These data are shown in Figure 4.

In this outbreak, the first 6 cases of pharyngitis developed in October as the result of the introduction into the community of a boy with an upper respiratory infection. In November, 10 more children contracted the infection: 9 in the control and one in the sulfonamide-treated group. During this month, one child receiving sulfanilamide became a "contact" carrier of the epidemic-inducing type. The outbreak subsided quickly: only 2 more children who were not receiving sulfonamide prophylaxis developed pharyngitis, one in December and one in January. Three more "contact" carriers appeared among the children in the sulfanilamide-treated group, respectively in December, February, and March. Only 2 "contact" carriers were observed in the control group: one began to harbor type 36 streptococci in December and another in January. The carrier rate of miscellaneous Group A streptococci dropped more precipitously in the control than in the sulfonamide group.

Comparison of the length of the carrier state in "post-infection" and "contact" carriers

During the course of the 4 major outbreaks of streptococcal pharyngitis, 120 children contracted clinical infections and 39 became "contact" carriers. Septic complications occurred in only 2 of the 120 patients: one boy developed cervical adenitis and another chronic sinusitis. Since it is well known that individuals with purulent 


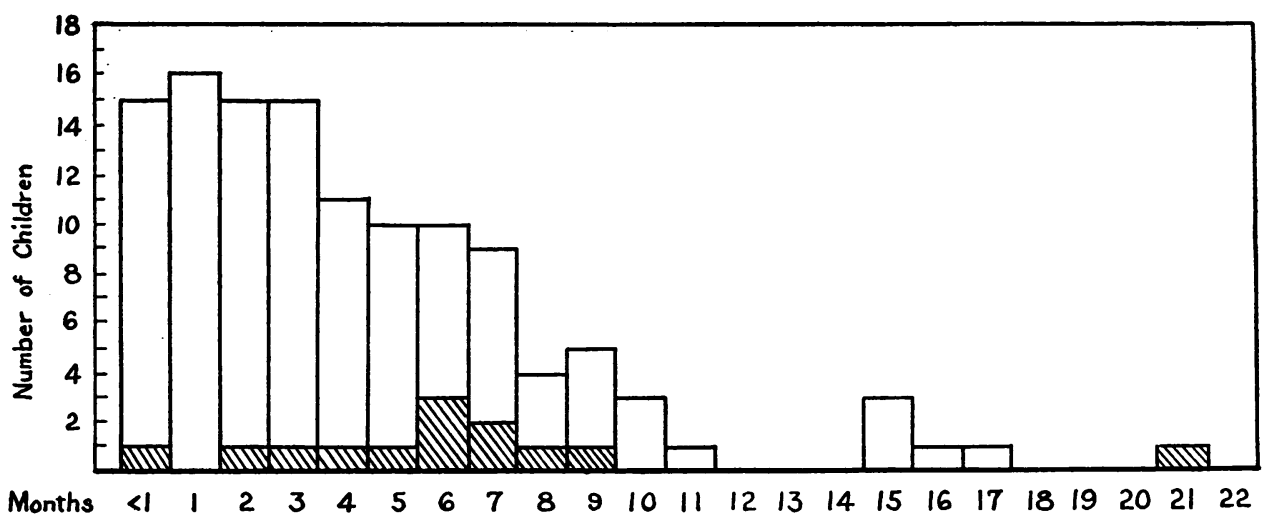

Fig. 5. One Hundred and Eighteen "Post-Infection" Carriers: Length of Carrier State (4 Major Outbreaks Combined) Shaded areas $=$ Individuals with tonsils

sequelae tend to harbor streptococci for long periods, these 2 boys were omitted from the comparison. Children in the sulfanilamide-treated groups, who became carriers of the epidemicinducing strains, were not included among the 39 "contact" carriers, because it seemed possible that in the absence of sulfonamide prophylaxis these individuals might have developed clinical symptoms.

The data on the length of the carrier state in 118 "post-infection" and in 39 "contact" carriers is presented in Figures 5 and 6.

In spite of the fact that 105 of the 118 "postinfection" carriers had had their tonsils removed, many of these individuals harbored the epidemicinducing strains for several months. The length of the carrier state in the "contact" was shorter

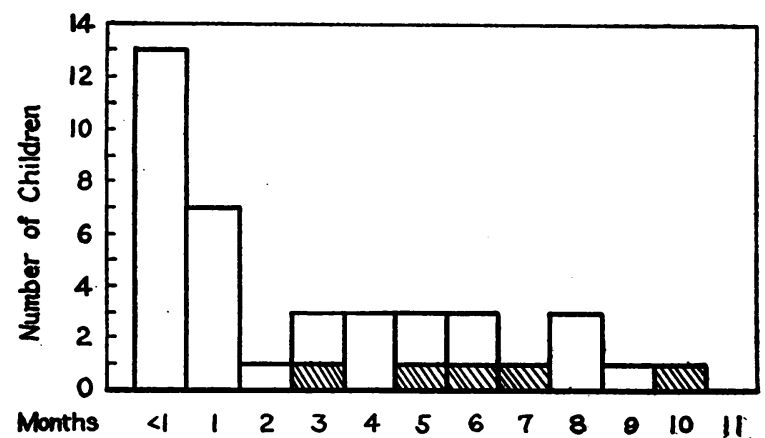

Fig. 6. Thirty-Nine "Contact" Carriers: Length of Carrier State (4 Major Outbreaks Combined; "Contact" Carriers Receiving Sulfonamide ProPHYLAXIS NOT INCLUDED)

Symbols same as in Figure 5. than in the "post-infection" carriers: over 50 per cent of the former compared with 29 per cent of the latter carried streptococci for one month or less. Only 5 of the 39 "contact" carriers retained their tonsils.

SUMMARY OF FINDINGS OBTAINED DURING THE 4 WINTERS 1938-42 (MAJOR OUTBREAKS)

During these 4 winters, when outbreaks of streptococcal pharyngitis due to Group A streptococci of single types (types $4,27,15$, and 36 , respectively) occurred, no rise in the carrier rate of the epidemic type preceded the appearance of cases. In the epidemics due to types 4 and 27, the incidence of "contact" carriers rose slowly and only reached a peak during the month when the greatest number of cases occurred. In the outbreaks due to type 15 and type 36 , in each instance, only 2 children among those who were not receiving sulfonamide prophylaxis became "contact" carriers after the epidemic had started.

With the exception of the epidemic due to type 36 streptococcus, when a temporary rise occurred during the first month of the outbreak, the carrier rates of miscellaneous Group A streptococci were falling at the time that the epidemics began.

B. Minor outbreaks of streptococcal upper respiratory infections due to the dissemination of types present in the community

The 2 winters $1937-38$ and $1942-43$ are alike in that no major outbreaks occurred. Strains 
carried by "healthy" carriers spread to other children who became "contact" carriers without developing evidence of infection, and subsequently, a few children developed pharyngitis due to these types.

1937-38. Weekly throat cultures were not taken routinely until January 1,1938 , and therefore no accurate data on the number of Group A carriers present in the community during the summer and fall of 1937 were available.

In May 1937, a boy carrying type 32 streptococcus was admitted. Five children became "contact" carriers of this type before the first case appeared the end of December. This infection spread slowly and did not reach a definite peak: during each of the 3 succeeding months (January, February, and March), 3 cases occurred, followed by one in April and another in May, making a total of 12 . The upper respiratory symptoms associated with this infection were mild. From the beginning of January until April, 9 more children became contact carriers of type 32 .

\section{Group $A$ streptococcus carriers in the community during the winter 1942-43}

During this winter the group included 71 "new" and 37 "old" patients. Seventeen "new" children were Group A streptococcus carriers. The following types were represented: types 1 , $2,14,17,18,19,41$, and 44, and 6 strains of undetermined types. By November 1, 7 of these 17 carriers no longer harbored hemolytic streptococci.

Type 19. Type 19, however, introduced by a boy admitted in July, showed a tendency to spread to other children. During the summer, 5 other boys became "contact" carriers of this type. In October, a girl developed pharyngitis due to this type; and one month later, a boy contracted this infection. Neither of these children developed rheumatic sequelae. No further cases of pharyngitis due to type 19 appeared although one "contact" carrier harbored this type until May and one "post-infection" carrier until June.

Four of the 37 "old" children were carriers of Group A streptococci. Two children carried type 36, the epidemic strain of the previous year. One "new" girl became a "contact" carrier of this strain in February and carried this type for 2 months. One child carried type 44, but this strain showed no tendency to spread to other children.

Type 37. One boy, admitted in November 1941, was an intermittent carrier of type 37 for more than one year. During the first winter of his stay, 1941-42, 2 children became temporary "contact" carriers of this type, but no cases of pharyngitis developed. During the second winter, 1942-43, 5 children again became "contact" carriers and 6 other children contracted upper respiratory infections due to this type. Two of the latter subsequently developed mild rheumatic manifestations.

SUMMARY OF FINDINGS OBTAINED DURING THE

2 WINTER SEASONS, 1937-38 AND 1942-43

(MINOR OUTBREAKS)

During both these winters, a relatively small number of cases of streptococcal pharyngitis occurred in the community. In each instance the infections were caused by the dissemination of types derived from known carriers who had been in the community for several months. These strains first spread to other children who became "contact" carriers. Subsequently, a few children developed pharyngitis due to these types. During 1937-38, 12 cases of pharyngitis were caused by type 32 streptococcus and during 1942-43, 2 cases by type 19 and 6 by type 37 .

\section{SUMMARY OF FINDINGS REGARDING "HEALTHY" OR ADMISSION CARRIERS}

During the 5-year period, 1938-43, when the weekly incidence of Group A carriers was determined, 102 of the 348 "new" children were

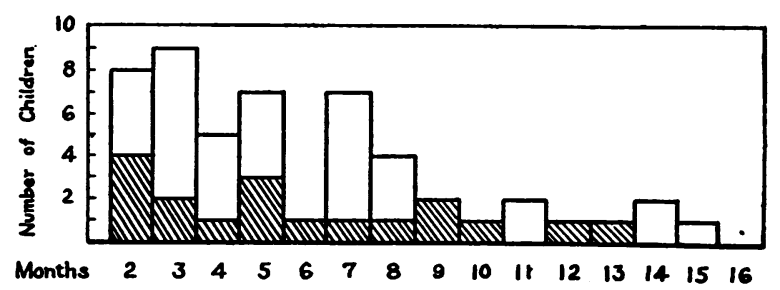

Fig. 7. Fifty-One Chronic "Healthy" or Admission Carriers: length of Carrier State (33, or $65^{\circ}$ per CENT, had Had Tonsillectomies)

Symbols same as in Figure 5. 
"healthy" carriers of Group A streptococci. Fifty-one or 50 per cent, of the 102 "healthy" carriers harbored streptococci for 2 months or longer while under observation. The length of the carrier state in these 51 individuals, the majority of whom had had their tonsils removed, is presented in Figure 7.

\section{DISCUSSION}

The incidence of outbreaks and cases of streptococcal pharyngitis and of Group A streptococcus carriers, observed in groups of rheumatic children during 6 successive winters, has been described. The relation of these upper respiratory infections to rheumatic relapses has been previously reported and, except in the case of the current year, 1942-43, is not discussed.

During each of 4 winter seasons when a fairly large number of streptococcal upper respiratory infections due to single types of Group A streptococci occurred in the community, a rise in the carrier rate of the epidemic-inducing types did not precede the appearance of the epidemics. These strains were introduced from outside sources and were not derived from Group A streptococcus carriers who had been in the community for any length of time.

During each summer, a fairly large proportion of the children admitted were "healthy" carriers of Group A streptococci. The majority of these individuals had had tonsillectomies. In most instances, these strains did not spread to other children, and usually disappeared from throat cultures by the beginning of the winter season (i.e., December 1). Some carriers, however, irrespective of whether or not their tonsils had been removed, continued to carry the same type for periods as long as 2 years, but, in spite of prolonged and intimate contact during the winter months, did not cause infection in other individuals.

Occasionally, however, types derived from carriers spread to other children who also became carriers; and subsequently, a few cases of pharyngitis due to these types appeared. In one instance, a single type of a Group A streptococcus (type 33) became widely disseminated but caused only one mild upper respiratory infection. Other types infected a small number of children and then failed to spread further.
It is well known that food-borne outbreaks of streptococcal upper respiratory infections are usually explosive and subside quickly. On the other hand, epidemics of streptococcal pharyngitis or scarlet fever, where the infection is transmitted from person to person, often last for weeks and months. This difference is undoubtedly related to the size of the infecting dose. Under ordinary conditions, only a small number of bacteria are transferred from one individual to another. It seems probable that, in many instances where individuals are in close contact, the number of streptococci necessary to cause symptoms may be acquired fractionally as suggested by Dudley. A considerable period of time may elapse before a sufficiently large dose is transferred, thus explaining the slow spread of the infection.

Aside from the size of the infecting dose, differences in susceptibility also play a part. In the presence of sporadic infections, when a few children in a ward develop streptoccal pharyngitis due to a single type and the rest remain uninfected, it seems probable that the latter group are relatively resistant to that particular type. Evien during major outbreaks, when the number of "post-infection" and "contact" carriers in the community was large, many children escaped infection. At the present time, our knowledge of streptococcal immunity is very limited; but in our experience, children usually do not contract pharyngitis due to the same type more than once. These observations suggest the existence or development of type-specific immunity.

The possibility that the infectivity of different types or variants of the same type of streptococci may vary from season to season and from year to year must also be considered. During each of the 4 years when major outbreaks of streptococcal upper respiratory infections occurred, a few children carrying the epidemic type of the previous winter remained in the institution. In spite of the fact that large groups of "new" children were admitted during the summer and fall months, in only one instance did the epidemic type of the previous year induce a few cases of pharyngitis among the "new" children. During the autumn of 1940,7 of the 70 newly admitted children developed mild upper respiratory infec- 
tions due to type 27, the type prevalent in the institution during the previous winter. The streptococci isolated from these 7 patients had smaller capsules than those isolated during the original outbreak and the colonies were matt rather than mucoid. It is also well known that strains isolated at the end of an outbreak often give less well defined serological reactions than those isolated at the beginning. This evidence suggests that the streptococci themselves tend to change during the course of epidemics.

Some investigators believe that individuals do not become carriers of Group A streptococci except as the result of infection but our experience is not in accord with this view. In 2 of the major outbreaks, due, respectively, to type 15 and type 36 streptococci, only a few children became "contact" carriers of the epidemicinducing strain. In the 2 outbreaks, due, respectively, to streptococci types 4 and 27, on the other hand, a large number of children were considered to be "contact" carriers. The spread of infection with types 4 and 27 was slower and the clinical symptoms less severe than with type 15 or type 36 . Our observations suggest that the incidence of "contact" carriers varies inversely with the virulence of the infecting strain. Thus, in the case of type 33 which became widely disseminated during the autumn of 1939, 22 children became "contact" carriers and only one child developed mild upper respiratory symptoms due to this type. This strain formed small matt colonies and no capsule was demonstrable in India Ink preparations.

There is no doubt that the virulence, not only of different types of Group A streptococci but also of different strains of the same type, may vary widely. Individuals sometimes become chronic carriers of streptococci belonging to groups other than Group A, groups rarely pathogenic for human beings. It seems possible therefore that Group A streptococci of low virulence or those belonging to types to which the individual is resistant may exist as saprophytes on the mucous membranes of the nasopharynx. At the present time, in our opinion, the conclusion that all "contact" carriers are really the result of infection is open to question.

Our findings that outbreaks of streptococcal pharyngitis are not necessarily preceeded by a rise in the carrier rate of the epidemic-inducing type are not in accord with those of most other investigators. The epidemics studied were small, but the data included the whole community and were not based on the selection of samples considered to be representative. The observations were limited to rheumatic children, and the susceptibility of the rheumatic individual to streptococcal infections may differ from that of the normal. The fact that, during each winter, the group of children was fairly isolated may also have influenced our findings. The majority of the infections were not severe; and none of the children developed scarlet fever. The possibility that some of our cases were so mild that these individuals might have been considered to be "healthy" carriers in communities with less medical supervision does not, however, explain the discrepancy between our observations and those of others. The symptoms developed by some of the patients at the beginning of each outbreak were sufficiently severe (temperatures of 102 to $104^{\circ} \mathrm{F}$.) so that they would not have been overlooked. In our opinion, major outbreaks of streptococcal pharyngitis are usually not caused by chronic Group A carriers in the community. The greatest source of danger is the introduction into the group of an individual with an active streptococcal infection, due to a type not previously present.

\section{SUMMARY}

1. Major and minor outbreaks as well as sporadic cases of streptococcal pharyngitis occurring in groups of rheumatic children in a sanatorium during a 6-year period are described.

a. Major outbreaks were due to Group A streptococci of a single type not previously present and were not preceded by a rise in carrier rate.

b. Minor outbreaks were preceded by a slow spread from carriers to other individuals without at first causing infection. Subsequently, a small number of clinical cases due to these types developed.

c. Sporadic cases arose directly from carriers and were not preceded by a dissemination of the streptococci to healthy individuals.

2. The length of the carrier state was studied. 
a. Twenty-nine per cent of the children admitted during the summer and fall months were carriers of Group A hemolytic streptococci. With few exceptions, these microorganisms did not spread to other individuals and disappeared after a few months.

b. The epidemic-inducing types of streptococci persisted longer in "post-infection" than in "contact" carriers.

c. The length of the carrier state was not related to the presence or absence of tonsils.

The authors are greatly indebted to Dr. Homer F. Swift of the Hospital of the Rockefeller Institute for Medical Research for assistance in the preparation of this manuscript.

\section{BIBLIOGRAPHY}

1. Lancefield, R. C., A serological differentiation of human and other groups of hemolytic streptococci. J. Exper. Med., 1933, 57, 571.

2. Lancefield, R. C., Antigenic complex of streptococcus haemolyticus; demonstration of type-specific substance in extracts of streptococcus haemolyticus. J. Exper. Med., 1928, 47, 91.

3. Griffith, F., The serological classification of streptococcus pyogenes. J. Hyg., 1934, 34, 542.
4. Bloomfield, A. L., and Felty, A. R., On the mode of spread of an outbreak of acute tonsillitis. Bull. Johns Hopkins Hosp., 1923, 34, 393.

5. Bloomfield, A. L., and Felty, A. R., Definition of hemolytic streptococcus parasitism in the upper air passages of healthy people. Arch. Int. Med., 1923, 32, 386.

6. Bloomfield, A. L., and Rantz, L. A., An outbreak of streptococcic septic sore throat in an army camp. J. A. M. A., 1943, 121, 315.

7. Dudley, S. F., The Schick Test, Diphtheria and Scarlet Fever. Med. Res. Counc. Special Report Series, No. 75, His Majesty's Stat. Off., London, 1923, p. 48.

8. Schwentker, F. F., Survey of hemolytic streptococci in certain army camps. Army M. Bull., 1943, 65, 94.

9. Kuttner, A. G., and Krumwiede, E., Observations on the effect of streptococcal upper respiratory infections on rheumatic children: A three year study. J. Clin. Invest., 1941, 20, 273.

10. Kuttner, A. G., and Reyersbach, G., The prevention of streptococcal upper respiratory infections and rheumatic recurrences in rheumatic children by the prophylactic use of sulfanilamide. J. Clin. Invest., 1943, 22, 77.

11. Krumwiede, E., A comparison of the value of the agglutination and precipitin reactions in the serological typing of Group A streptococci. J. Bact., 1943, 46, 117. 